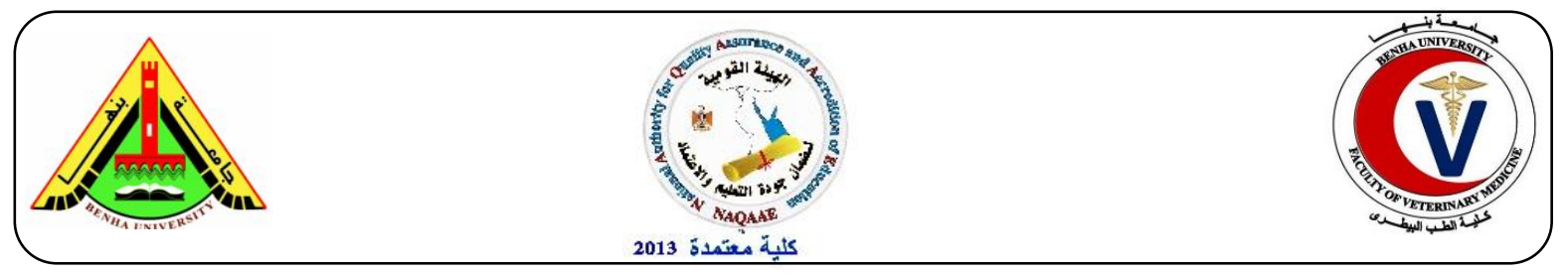

\title{
The protective effect of ginger extract on induced fatty liver in Albino rats: Histological and biochemical study
}

\section{Mahmoud A Emam 1, Fatma A Gad ${ }^{2}$, Badia Abugherin ${ }^{3}$}

${ }^{1}$ Histology, ${ }^{2}$ Clinical Pathology Departments, Faculty of Veterinary Medicine, Benha University, Egypt. ${ }^{3}$ Anatomy and Histology Department, Faculty of Veterinary Medicine, Tripoli University, Libya

\section{A B S T R A C T}

The aim of the current study is to evaluate the hepatoprotective effect of ginger extract on induced fatty liver in rats. A total of 20 Albino rats (10-12 weeks old and 160-220 g body weight) were used for this study. The rats were divided into 4 groups ( 5 rats/ group). Group I was administrated distilled water, group II was fed high fat diet (HFD), group III was administrated ginger extract and group IV were orally administrated ginger extract 2 weeks earlier before HFD and then received both ginger and HFD for 10 weeks. At the end of the experiment, blood samples were collected for biochemical analysis. Also, the livers from all animals were collected for histological examination. Biochemical analysis revealed significant increases in serum levels of ALT, AST, TGs and TC in rats from group II compared to other groups and its liver showed many areas abnormal features of hepatocytes with fatty changes and apoptotic signs with pycnotic nuclei. However, those from group IV showed improvement of biochemical and histological pictures. This study concluded that ginger extract ameliorates the biochemical and histological changes associated with induced fatty liver in Albino rats.

Keywords: Histology, Biochemical analysis, Fatty liver, Ginger extract, Rat

\section{INTRODUCTION}

Fatty liver is a common condition that is considered as manifestation of obesity and metabolic syndrome (Pango et al., 2002). Histologically, it is characterized by deposition of lipid in the cytoplasm of hepatocytes (Ratziu et al., 2010). Fatty liver is markedly increased in Egyptian people throughout last years (Helal et al., 2012). Medicinal plants are natural herbs that are free from side effects and available. Nowadays, they are the symbol of safety in contrast to the synthetic drugs which are unsafe.

Ginger (Zingiber officinale Roscoe) is cultivated for medicinal and culinary purposes (Baliga et al., 2011). Ginger contains several valuable compounds. Its high antioxidant value has proved highly effective with its ability to scavenge a number of free radicals and protect cell membrane lipids from oxidation in a dose 
dependent manner. Ginger is a very famous drink used all over the world. Traditionally, it is used for flu relief, general sickness, gastrointestinal issues, and immune system support (Ali et al., 2008; Baliga et al., 2011). Moreover, ginger extract has been reported to exert its anti-hyperlipidemic effects by decreasing serum levels of total cholesterol and triglycerides (Ahmida and Abuzogaya, 2009). Recently, researchers are saying that ginger supplementation may have the potential to treat people who suffer from fatty liver disease (Mallikarjuna et al., 2008; Shati and Elsaid, 2009; Sahebkar, 2011 and Rahimlou et al., 2016). Ginger has been shown to be a hepatoprotective and hardly there are any reports of toxic effects of ginger (Haniadka et al., 2013). Therefore, this study aimed to assess the protective effect of ginger extract on the induced fatty liver in Albino rats via biochemical and histological analysis.

\section{MATERIALS AND METHODS}

2.1. Preparation of ginger extract Ginger extract was prepared by peeling ginger and slicing it thinly to maximize the surface area. Then, $50 \mathrm{gm}$ of peeled ginger was boiled in liter of water for at least 15 minutes. After filtration, the extract was kept at $4{ }^{\circ} \mathrm{C}$, prepared and given daily using gastric tube at dosage rate of $125 \mathrm{mg} / \mathrm{kg}$ body weight (b.wt) to rats according to Helal et al (2012).

\subsection{Experimental design}

A total of twenty male Albino rats of 10-12 weeks old and 160-220 g b.wt. were obtained for this study. They were housed in separate metal cages and kept at a constant environmental and nutritional condition throughout the period of the experiment. All animals were left for acclimatization before the beginning of the experiment. The rats were divided into 4 groups (5 rats/ group). Group I was orally administrated distilled water, group II was fed high fat diet (HFD) containing $17 \mathrm{~g}$ fat plus $3 \mathrm{~g}$ corn oil/ $100 \mathrm{~g}$ diet with $1 \%$ cholesterol powder and $1 \%$ cholic acid powder according to Kim and Park (2008), Abozaid et al., (2012) and Sampathkumar et al., (2011) respectively for 10 weeks, group III was orally administrated ginger tea extract $(125 \mathrm{mg} / \mathrm{kg})$ daily by gastric tube according to Helal et al., (2012), and group IV were orally administrated ginger extract 2 weeks earlier before HFD and then received both ginger and HFD for 10 weeks.

\subsection{Blood samples}

Blood samples from retro-orbital plexuses were collected after overnight fasting from all animal groups at the end of experiment. Sera were separated after centrifuging at $1800 \mathrm{rpm}$ for 10 minutes then aliquot and stored at -20C until examined.

\subsection{Biochemical analysis}

Serum alanine amino transferase (ALT) and aspartate aminotransferase (AST) were determined according to the methods of Gella et al., (1985). Also triglycerides (TG) and total cholesterol (TC) were measured according to Fossati and Prencie (1982) and Flegg (1973) respectively.

\subsection{Collection of tissue specimens}

Rats from all groups were sacrificed by decapitation. The livers were rapidly collected, cut into small pieces and immersed in $10 \%$ neutral formalin for 72 hr. Then, dehydrated, cleared, embedded in paraffin, sectioned and stained with hematoxylin and eosin (H\&E). The staining method was according to Bancroft and Gambel (2002). 


\subsection{Statistical Analysis:}

Statistical analysis was by one-way analysis of variance (ANOVA). If one-way ANOVA indicated a significant difference, then differences between individual groups were estimated using Duncan as a post hoc. Results are expressed as the mean \pm standard error of mean. A $P$-value of less than 0.05 was considered significant.

\section{RESULTS}

3.1. Biochemical effect of ginger extract on serum ALT, AST, TGs and TC

As show in table (1), there were significant increases in the activities of ALT, AST and serum levels of TGs and TC in group II compared to other groups. However, group IV revealed significant decreases in ALT and AST activities and in TGs and TC levels when compared with those in group II. Meanwhile, ginger treated-group showed non-significant changed in the all measured parameters when compared with control group.

\subsection{Histological observations}

Sections of livers from group I showed normal and classic histological structure. Hepatocytes were polygonal and had vesicular nuclei. The hepatic cords were radiating from the central veins and the blood sinusoids and their von Kupffer cells

Table (1): Mean \pm SE values of ALT, AST, TGs and TC of all groups

Different superscripts ( $\mathrm{a}, \mathrm{b}$ and $\mathrm{c}$ ) in the same column indicate significant difference at $p \leq 0.05$

\begin{tabular}{lcccc}
\hline Groups & ALT $(\mathrm{U} / / \mathrm{L})$ & TST(U/L) & TG $(\mathrm{gm} / \mathrm{dl})$ & TC $(\mathrm{gm} / \mathrm{dl})$ \\
\hline Group I & $28.14 \pm .09^{\mathrm{a}}$ & $51.44 \pm 0.72^{\mathrm{a}}$ & $91.67 \pm 10.26^{\mathrm{a}}$ & $60.57 \pm 2.89^{\mathrm{a}}$ \\
Group II & $62.00 \pm 1.06^{\mathrm{c}}$ & $83.35 \pm 1.32^{\mathrm{c}}$ & $204.87 \pm 5.04^{\mathrm{c}}$ & $125.17 \pm 3.26^{\mathrm{c}}$ \\
Group III & $29.33 \pm .16^{\mathrm{a}}$ & $49.94 \pm 0.50^{\mathrm{a}}$ & $90.01 \pm 9.76^{\mathrm{a}}$ & $61.67 \pm 1.26^{\mathrm{a}}$ \\
Group IV & $37.00 \pm .2^{\mathrm{b}}$ & $62.54 \pm 0.91^{\mathrm{b}}$ & $151.00 \pm 3.07^{\mathrm{b}}$ & $92.27 \pm 1.96^{\mathrm{b}}$ \\
\hline
\end{tabular}

were seen among the cords (Fig. 1). Meanwhile, liver sections from group II revealed many areas abnormal features of hepatocytes with fatty changes (Fig. 2A). Most of hepatocytes showed ballooning (Fig. 2B). Moreover, numerous minute cytoplasmic lipid droplets with centrally placed nuclei (Fig. 2B) and well-defined a large fat droplet displacing the nucleus to the cell periphery were observed (Fig. 2D). Also, apoptotic signs with pycnotic nuclei appeared in some sections (Fig. 2C). Sections of liver from group III showed nearly normal histological architecture like those of group I (Fig. 3). Liver sections from group IV showed a marked decrease of fatty changes (Fig. 4A) compared with positive control rats (Fig. 2). However, some sections still showed fatty changes with few scattered fat cells (Fig. 4B) and others showed hydropic degeneration (Fig. 4C). 
The protective effect of ginger extract on induced fatty liver in Albino rats: Histological and biochemical study

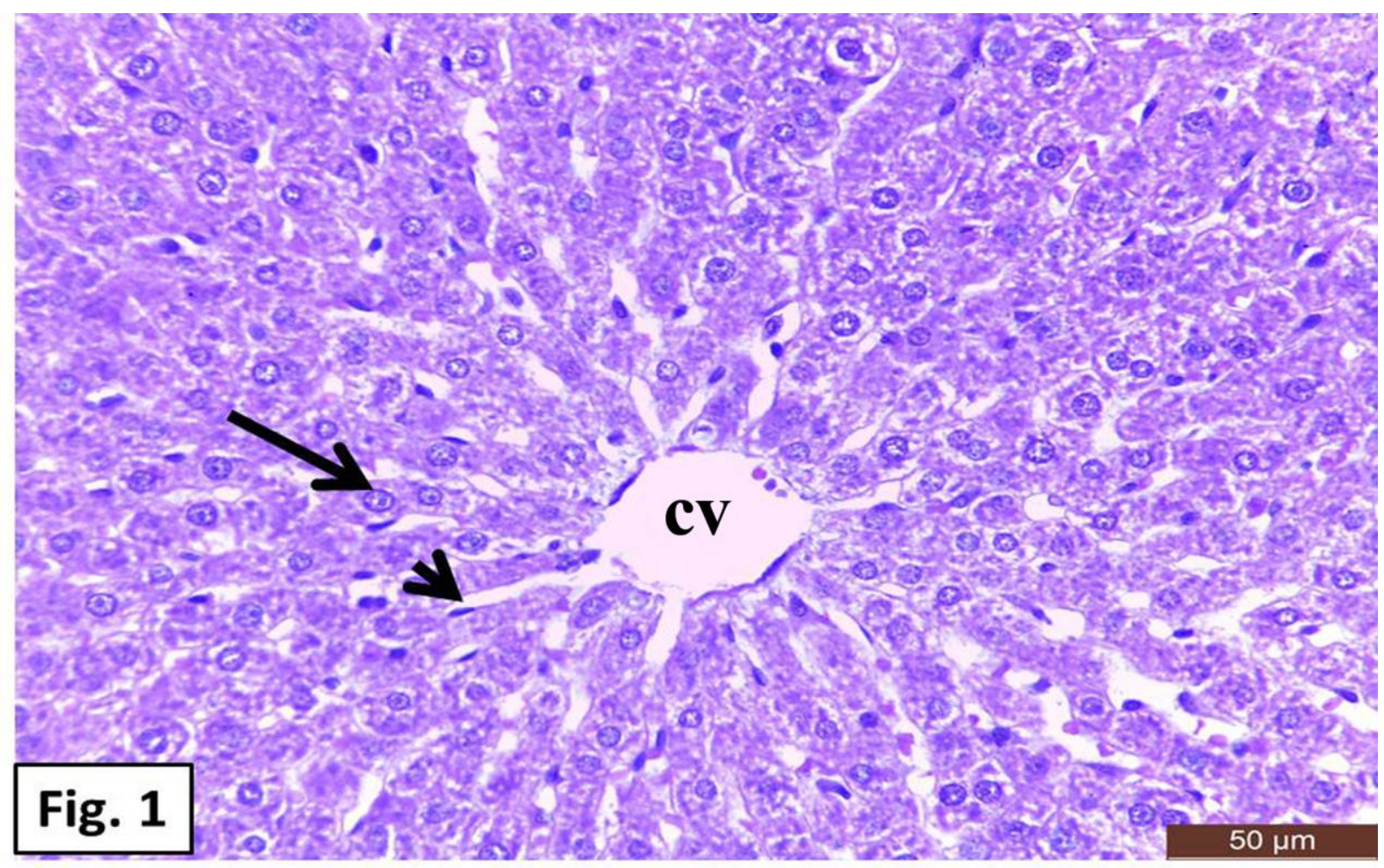

Fig. 1: Section of liver from rats of group I showing classic histological liver structure. Hepatocytes were polygonal and had vesicular nuclei (arrow). The hepatic cords were radiating from the central veins (CV) and the blood sinusoids and their von Kupffer cells (arrowhead) were seen among the cords. H\&E stain. Scale bar $=50 \mu \mathrm{m}$. 

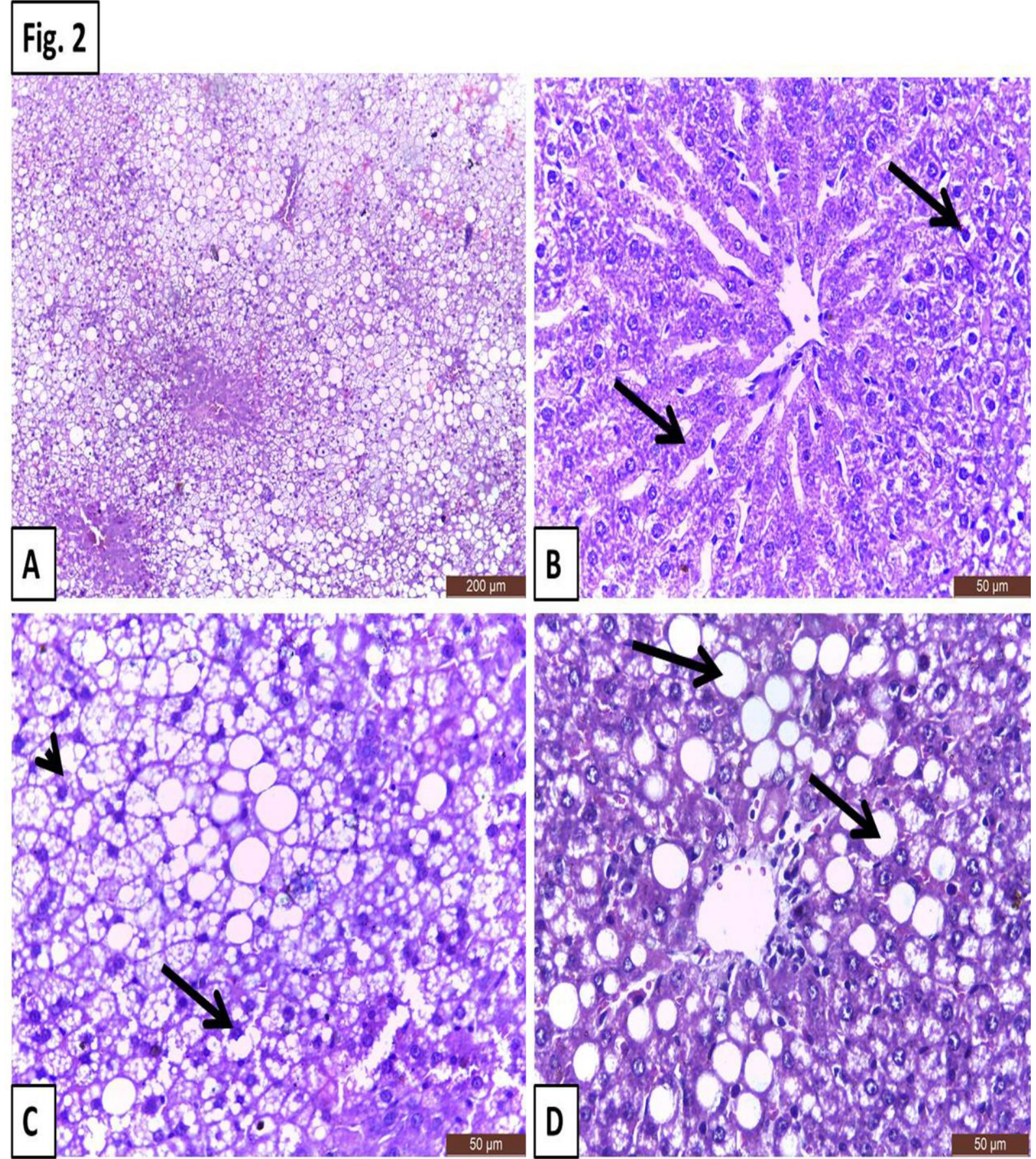

Fig. 2: Sections of livers from rats of group II. A: Photo showed many areas abnormal features of hepatocytes with fatty changes. B: Photo showed ballooning of hepatocytes (arrows). C: Photo showed numerous minute cytoplasmic lipid droplets in the hepatocytes with centrally placed nuclei (arrowhead) and apoptotic signs with pycnotic nuclei (arrow). D: Photo showed hepatocytes with well-defined a large fat droplet displacing the nucleus to the cell periphery (arrows). H\&E stain. Scale bars $(A=200 \mu \mathrm{m}, \mathrm{B}-\mathrm{D}=50 \mu \mathrm{m})$. 
The protective effect of ginger extract on induced fatty liver in Albino rats: Histological and biochemical study

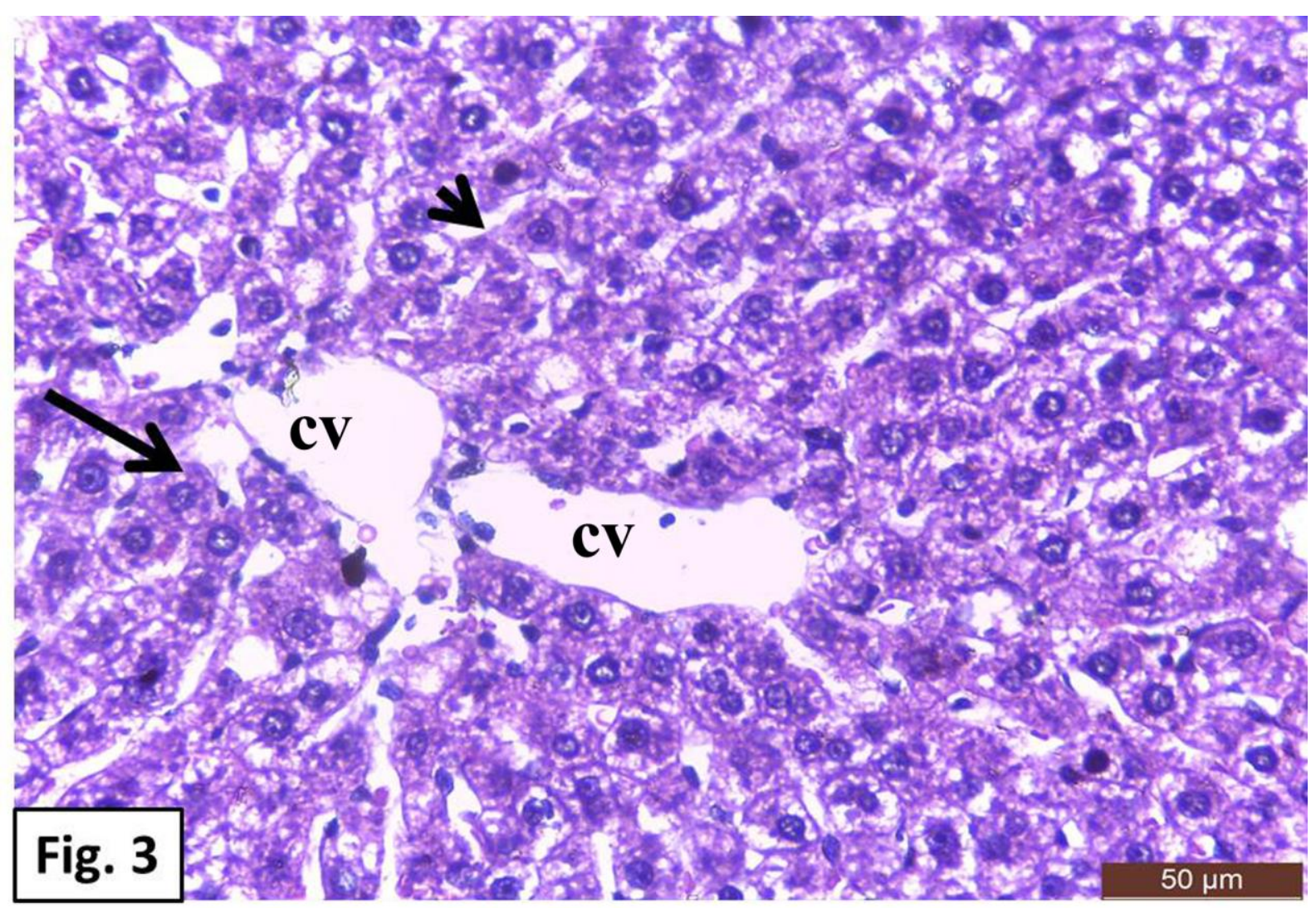

Fig. 3: Section of liver from group III showed nearly normal histological architecture like those of group I (Fig. 1). H\&E stain. Scale bar $=50 \mu \mathrm{m}$. 


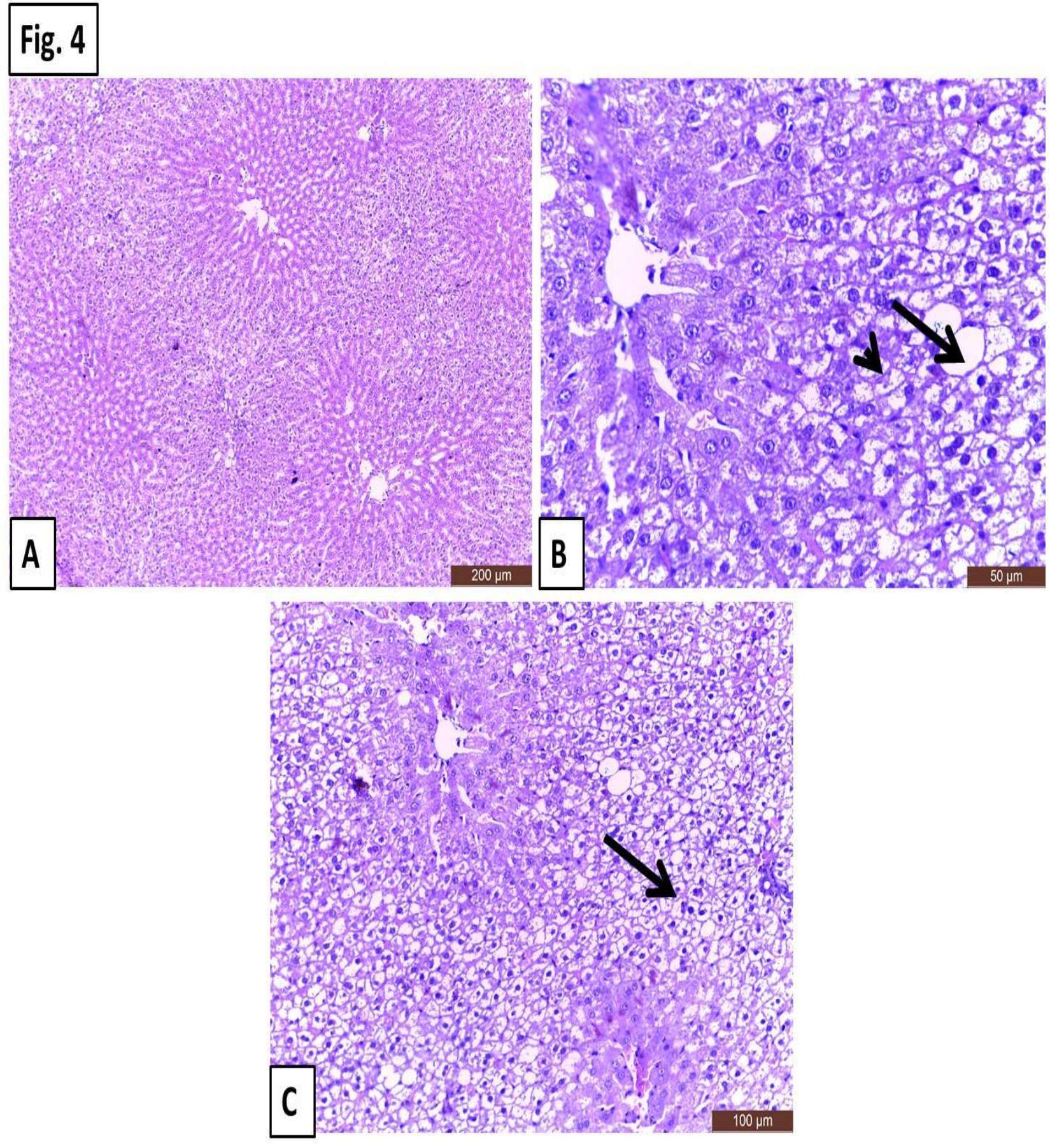

Fig. 4: Sections of livers from group IV. A: Photo showed a marked decrease of fatty changes compared with positive control rats (Fig. 2). B: Photo showed showed fatty changes (arrowhead) with few scattered fat cells (arrow). C: Photo showed hydropic degeneration (arrow). H\&E stain. Scale bars $(A=200 \mu \mathrm{m}, \mathrm{B}=50 \mu \mathrm{m}$ and $\mathrm{C}=100 \mu \mathrm{m})$.

\section{DISCUSSION}

The accumulation of triglycerides in hepatocytes is defined as fatty liver disease (Kleiner et al., 2005). Fatty liver is a major and common liver disease worldwide (Targher et al., 2010) and it increases the risks for mortality (Adams et al., 2005).The pathological picture of fatty liver varies from presence of small cytoplasmic fat droplets in hepatocytes (Szczepaniak et al., 2005), hepatocyte ballooning, apoptosis, inflammatory 
infiltrates, collagen deposition and finally to liver cirrhosis (Targher and Arcaro, 2007).

The present study was conducted to evaluate the protective effect of ginger extract on induced fatty liver in albino rats. Ginger's antioxidant properties and its triglyceride and cholesterol lowering effects were reported by Helal et al., (2012). Previously preclinical studies have shown that feeding rats a diet containing ginger $(1 \%)$ for four consecutive weeks was effective in ameliorating the hepatotoxic effects (Ajith et al., 2007 and Mallikarjuna et al., 2008). Moreover, Bhandari et al. (2003) recoded comparable beneficial effects of ginger than clinically used hepatoprotective drug silymarin.

In the current study, the administration of ginger extract as a protective agent before induction of fatty liver was recorded significant change in serum ALT, AST, TGs and TC levels when compared with fatty liver group indicating the ameliorative effect of ginger tea on the induced fatty liver in rats. ALT and AST Activities are good markers of assessment liver function. These enzymes are normally present in the cytoplasm of hepatocytes. When hepatocytes are damaged and or the permeability of hepatocytes increased, the enzymes released into the plasma (Atta et al., 2010). Rats fed HFD for 10 weeks showed fatty liver, necrosis and inflammation. These histological changes were associated with an increase in activities of serum ALT and AST. This is in agreement with Asha et al. (2007), who reported that, this significant increase may be due to rise in free radicals with decrease in the antioxidant enzyme levels. The significant decrease in serum ALT and AST in ginger administrated group supports the hepatoprotective effect of ginger that was inconsistence with Ajith et al., 2007; Yaccout et al., (2007); Helal et al., (2012) and Haniadka et al., (2013).

The ameliorative effect of ginger on TGs and $\mathrm{TC}$ in the present study is in agreement with Bhandari et al., (2005) and Fuhrman et al., (2000) who revealed that, ginger produces significant decrease in serum TGs and TC levels and exhibits a significant lipid lowering activity and protect the tissues from lipid peroxidation. Also, Matsuda et al. (2009) reported that supplementation of ginger to highcholesterol diet significantly reduced fat accumulation.

Ajith et al., (2007) owed the ameliorative role of ginger on the measured biochemical parameters to the antioxidant, antiinflammatory and free radical scavenging property of the ginger that scavenge the free radicals, minimize lipid peroxidation and prevent membrane damage and leakage of enzymes.

For the histological findings of the present study, the classic histological picture of livers from group I (negative control) was similar to finding of Helal et al. (2012). The finding of the livers from group II revealed an abnormal architecture in comparison to normal livers of group I. Many areas of fatty changes in hepatocytes such as an intracytoplasmic single fat droplet or many cytoplasmic minute lipid droplets were observed that was similar to Brunt et al. (2009). The hepatic damage ranged from the ballooning of the hepatocytes to replacement of the hepatocytes by complete fat cells (Helal et al., 2012).

From the current study we can conclude that the administration of ginger extract ameliorated the fatty changes in the livers 
of rats where it caused a marked decrease in lipid accumulation in hepatocytes compared with positive control rats.

\section{REFERENCES:}

Abozaid, O.A.R.; Mohamed, R.R.H. and Aml, H.M. 2012. Endothelial dysfunction in hypercholestrolemic rats' effect of Bezafibrate on endothelium derived substances. Benha Veterinary Medical Journal, 23(2): 24-33.

Adams, L.A.; Lymp, J.F.; Sauver, J.; Sanderson, S.O.; Lindor, K.D.; Feldstein, A. and Angulo, P. 2005. The natural history of nonalcoholic fatty liver disease: a populationbased cohort study. Gastroenterology, 129(1): 113-121.

Ahmida, M.H. and Abuzogaya, M.H. 2009. The effects of oral administration of green tea and ginger extracts on serum and hepatic lipid content in rats fed a hyperlipidemic diet. J Appl Sci Res, 5: 1709-1713.

Ajith, T.; Hema, U. and Aswathy, M. 2007. Zingiber officinale Roscoe prevents acetaminophen-induced acute hepatotoxicity by enhancing hepatic antioxidant status. Food Chem. Toxicol. 45: 2267-2272.

Ali, B.H.; Blunden, G.; Tanira, M.O. and Nemmar, A. 2008. Some phytochemical, pharmacological and toxicological properties of ginger (Zingiber officinale Roscoe): A review of recent research. Food Chem Toxicol, 46: 409-420.

Asha, K.; Snkar, T. and Viswanthan, P. 2007. Effect of tetracycline on pancreas and liver function of adult male albino rats. J.Pharma. Pharmacol, 59: 1241-1248.

Atta, A.; Elkoly, T.; Mouneir, S.; Gehan, K.; Alwabe, I. and Shaimaa, Z. 2010. Hepatoprotective effect of methanol extract of Zingiber officinale and Cichorium intybus. Indian Journal of Pharmaceutical Sciences, 72: 564-570.

Baliga, M.S.; Haniadka, R.; Pereira, M.M.; D'Souza, J.J. and Pallaty, P.L. 2011. Update on the chemopreventive effects of ginger and its phytochemicals. Crit Rev Food Sci Nutr, 51: 499-523.

Bancroft, J.D. and Gambel, M. 2002. Theory and Practice of Histological techniques, 5th edn. London, New York and Philadelphia: Churchill Livingstone.

Bhandari, U.; Kanojia, R. and Pillai, K. 2005. Effect of ethanolic extract of Zingiber officinale on dyslipidaemia in diabetic rats. J. Ethnopharmacol, 97: 227-230.

Bhandari, U.; Shamsher, A.; Pillai, K.K. and Khan, M.S.Y. 2003. Antihepatotoxic activity of ginger ethanol extract in rats. Pharm Biol, 41:68-71.

Brunt, E.M. and Tiniakos, D.G. 2009. Alcoholic and non alcoholic fatty liver disease . In Odze RD, Gold blum JR, eds, editors. Surgical pathology of the GI tract, liver , billary tract and pancreas.2nd Philadelphia: Elsevier.

Flegg, H.M. 1973. Quantitative-enzymaticcolourimeteric determination of total and HDL-C in serum or plasma. Annals of Clinical Biochemistry, 10: 79.

Fossati, P. and Prencie, L. 1982. Serum triglycerides

determined 
colorimeterically with an enzyme that produces hydrogen peroxide. Clin Chem, 28:2077-2080.

Fuhrman, B.; Rosenblat, M.; Hayek, T.; Coleman, R. and Aviram, M. 2000 Ginger Extract Consumption Reduces Plasma Cholesterol, Inhibits LDL Oxidation and Attenuates Development of Atherosclerosis in Atherosclerotic, Apolipoprotein E-Deficient Mice. The Journal of Nutrition, 130(5): 1124-1131.

Gella, F.J.; Olivella, T.; Cruz Pastor, M.; Arenas, J.; Moreno, R.; Durban, R. and Gomez, J.A. 1985. A simple procedure for routine determination of aspartate aminotransferase and alanine aminotransferase with pyridoxal phosphate. Cli Chem Cta, 153:241-247.

Haniadka, R.; Saxena, A.; Shivashankara, A.; Fayad, R.; Palatty, P.; Nazreth, N.; Francis, A.; Arora, R. and Baliga, M. 2013. Ginger Protects the Liver against the Toxic Effects of Xenobiotic Compounds: Preclinical Observations. J Nutr Food Sci, 3:226.

Helal, E.; Abd El-Wahab, S.; Sharaf, A. and Zedan, G. 2012. Effect of Zingiber officinale on fatty liver induced by oxytetracycline in albino rats. The Egyptian Journal of Hospital Medicine, 46: 26-42.

Kim, Y.J. and Park, T. 2008. Gense are differentially expressed in the epididymal fat of rats rendered obese by a high fat diet. Nut. R., 28: 414-422.

Kleiner, D.E.; Brunt, E.M.; Van Natta, M.; Behling, C.; Contos, M.J. Cummings, O.W. 2005. Design and validation of a histological scoring system for non-alcoholic fatty liver disease. Hepatology, 41:1313-1321. Mallikarjuna, K.; Sahitya Chetan, P.; Sathyavelu Reddy, K. and Rajendra, W. 2008. Ethanol toxicity: rehabilitation of hepatic antioxidant defense system with dietary ginger. Fitoterapia, 79:174178.

Matsuda, A.; Wang, Z.; Takahashi, S.; Tokuda, T.; Miura, N. and Hasegawa, J. 2009. Upregulation of mRNA of retinoid binding protein and fatty acid binding protein by cholesterol enriched-diet and effect of ginger on lipid metabolism. Life Sci, 84 (25-26):903-907.

Pango, G.; Pacini, G.; Gambino, R.; Mecca, F. and Depetris, N. 2002. Non alcoholic steatohepatitis insulin resistance and metabolic syndrom: further evidence for an etiologic association. Hepatology, 35:367-372.

Rahimlou, M.; Yari, Z.; Hekmatdoost, A.; Alavian, S.M. and Keshavarz, S.A. 2016. Ginger Supplementation in Nonalcoholic Fatty Liver Disease: A Randomized, Double-Blind, Placebo-Controlled Pilot Study. Hepat Mon, 16(1): e34897.

Ratziu, V.; Bellentani, S.; Cortez-Pinto, H.; Day, C. and Marchesini, G. 2010. A position statement on NAFLD/NASH based on the EASL 2009 special conference. Journal of Hepatology, 53(2): 372-384.

Sahebkar, A. 2011. Potential efficacy of ginger as a natural supplement for nonalcoholic fatty liver disease. World J Gastroenterol, 17(2):271272. 
Sampathkumar, M.T.; Kasetti, R.B.; Nabi, S.A.; Sudarshan, P.R.; Swapna, S. and Apparao, C. 2011. Antihyperlipidemic and antiatherogenic activities of Terminalia Pallida linn fruits in high fat diet-induced hyperlipidemic rats. J. Pharm Bioallied sci, 3(3): 449-452.

Shati, A.A. and Elsaid, F.G. 2009. Effects of water extracts of thyme (Thymus vulgaris) and ginger (Zingiber officinale Roscoe) on alcohol abuse. Food Chem Toxicol, 47:1945-1949.

Szczepaniak, L.S.; Nurenberg, P. and Leonard, D. 2005. Magnetic resonance spectroscopy to measure hepatic triglyceride content: prevalence of hepatic steatosis in the general population. American Journal of Physiology Endocrinology and Metabolism, 288 (2):462-468.
Targher, G. and Arcaro, G. 2007. Nonalcoholic fatty liver disease and increased risk of cardiovascular disease.

Atherosclerosis, 191(2):235-240.

Targher, G.; Day, C.P. and Bonora, E. 2010. Risk of cardiovascular disease in patients with nonalcoholic fatty liver disease. New England Journal of Medicine, 363(14):1341-1350.

Yaccout, G.; El-Guindy, N. and El-Azab, E. 2007. Amelioretic effect of Zingiber officinale on experimentally induced liver fibrosis in rats. J Med Res Instit, 28(2):154-159. 